\title{
ON UNIFORM SPACES AND TOPOLOGICAL ALGEBRA
}

\author{
G. K. KALISCH
}

There are several papers which deal with some generalization of metric spaces, for instance, Price [1], ${ }^{1}$ Krull's general valuation theory [2], Hyers' pseudonorm [3], and so on. We show in the present paper that spaces possessing a generalized metric (that is, the distance is an element of a partially ordered abelian vector group) are uniform spaces, and that, conversely, any uniform space is a generalized metric space. We also show that topological groups possess right invariant generalized metrics, and, therefore, topological linear spaces, rings, and fields have generalized norms. Although it would be possible to construct a theory based on entirely arbitrary partially ordered abelian semigroups, in view of the construction used in the proof of Theorem 2, it is sufficient to consider only vector groups whose components are real numbers and which are ordered lexicographically. This restriction is not very serious, however, as can be seen from [4] and [5].

The elements of the vector groups we are considering are real numbers indexed according to a partially ordered directed set $I$. Let $i, j, k$ be elements of $I$, then:

I (1) If $i \leqq j, j \leqq i$ then $i=j$.

(2) If $i \leqq j, j \leqq k$ then $i \leqq k$.

(3) Given $i$ and $j$ there exists $k$ such that $k \geqq i, k \geqq j$.

We shall denote the elements of $G$, our vector group, by $\left(r_{i}\right), r_{i}$ a real number, $i$ an element of $I$. We define the order relation in $G$ as follows: $g=\left(r_{i}\right) \leqq g^{\prime}=\left(r_{i}^{\prime}\right)$ in case, if $r_{j}>r_{j}^{\prime}$ for some $j$, there is some $k$ such that $k<j, r_{k}<r_{k}^{\prime}$, and $r_{m} \leqq r_{m}^{\prime}$ for all $m<k$. A space will be called a generalized metric space in case there is given a function $(s, t)$ defined on $S \times S$ with $s \in S, t \in S$, with values in a partially ordered vector group $G$ as described above, and satisfying the following:

II (1) $(s, t) \geqq 0 ;(s, t)=0$ if and only if $s=t$.

(2) $(s, t)=(t, s)$.

(3) $(s, t) \leqq(s, r)+(r, t)$.

A generalized metric space will be a topological space, and the generalized metric will define a uniform structure in $S$ in case we define neighborhoods or convergence properly. Owing to the fact that $G$ is partially ordered it is possible to topologize $G$ by defining neighbor-

Received by the editors May 16, 1946, and, in revised form, June 4, 1946.

1 Numbers in brackets refer to the bibliography at the end of the paper. 
hoods of 0 in $G$ (weak case) by means of the vectorial structure of $G$; in terms of convergence it means that a set of vectors converges to a limit vector in case all components converge to the corresponding components of the limit vector (convergence of directed sequences). The corresponding neighborhoods of 0 are subsets of $G$ depending on a finite set $i_{1}, \cdots, i_{n}$ of elements of $I$, and a positive real number $\epsilon$ as follows:

$$
N_{i_{1}}, \ldots, i_{n} ; \epsilon(0)=\left[c ;\left|c_{i_{k}}\right|<\epsilon ; k=1, \cdots, n\right]
$$

where $c_{i}$ is the $i$-component of $c$. As soon as there is defined in $G$ a topology it is possible to topologize and uniformize $S$ : a neighborhood $U_{N}(s)$ for $s \in S$ and $N$ a neighborhood of 0 in $G$ is defined as

$$
U_{N}(s)=[t ;(s, t) \in N] .
$$

Since we wish to show that every uniform space is a generalized metric space, and since uniform spaces are subspaces of direct products of metric spaces (cf. [6]), it is necessary to use the weak topology of $G$. The topology and uniform structure of $S$ can also be given in terms of convergence of directed sequences of elements of $G$ : a directed sequence $\left(s_{i}\right)$ of elements of $S$ converges to $s \in S$ in case $\left(s, s_{i}\right)$ converges to $0 \in G$. We have the following theorem.

Theorem 1. A generalized metric space is a uniform space.

The most convenient formulation of uniform spaces for the purpose of this theorem is the one on page 7 of [6].

Conversely, let the uniform space $S$ be isomorphic to a subset of the direct product $P=\prod P_{\mu}$ where $P_{\mu}$ are metric spaces and the $\mu$ 's range over a set $M$. Let $M_{0}$ be the set of all finite subsets of $M$. The generalized distance between $s$ and $t$ for $s$ and $t$ in $S$ is then defined by $(s, t)=\left(r_{m}\right)$ for $m \in M_{0}, r_{m}=\max _{\mu \in m}$ (dist $\left.\left(s_{\mu}, t_{\mu}\right)\right)$ where $s_{\mu}$ is the component of $s$ in $P_{\mu}$ and dist $\left(s_{\mu}, t_{\mu}\right)$ is the (ordinary) distance between $s_{\mu}$ and $t_{\mu}$ in $P_{\mu}$ and $\left(r_{m}\right)$ is an element of the group $G$ described above. This definition of $(s, t)$ makes $S$ a generalized metric space isomorphic with the original one. Thus we have the following theorem.

THEOREM 2. Every uniform space is isomorphic to a generalized metric space.

If $P$ has only a finite number of noncompact direct factors then a strong topology can be used in $G$.

Topological groups are uniform spaces and hence generalized metric spaces as well. Kakutani's result on right invariant metrics in 
topological groups [7] suggests that the generalized metric in question can be chosen so as to be right (left) invariant, that is, $(x a, x b)$ $=(a, b)$ for all $x$ in the topological group $S$. We can apply Kakutani's proof to the individual components of the generalized metric of $S$ each of which constitutes a pseudometric (nonseparated metric) of $S$. We can thus introduce a right (left) invariant generalized metric into $S$ topologically equivalent to the one originally given, because the replacement of each component of a generalized metric by a topologically equivalent pseudometric does not change the topology of the generalized metric space. We therefore have the following theorem.

THEOREM 3. Every topological group has a right (left) invariant generalized metric, and, hence, topological linear spaces, rings, and fields possess generalized norms.

By a linear space we mean, of course, an abelian group with a topological field $F$ as coefficient domain satisfying the obvious continuity conditions; and a generalized norm is a function $|t|$ defined for $t$ in the algebraic structure $T$ in question with values in a partially ordered abelian group $G$ as above with the following properties:

III (1) $|a| \geqq 0 ;|a|=0$ if and only if $a=0$.

(2) $|a+b| \leqq|a|+|b|$.

$\left(3_{L}\right) \quad \alpha a \mid$ is a continuous function of $a$ and $\alpha \in F$.

$\left(3_{R}\right)|a b|$ is a continuous function of $a$ and $b$.

Here 0 is the identity element of $G, O$ the identity element of $T$; $a$ and $b$ are in $T$. III $\left(3_{L}\right)$ is valid for topological linear spaces, while III $\left(3_{R}\right)$ is valid for topological rings and fields. The results here mentioned are in a sense the best possible, as can be seen by the fact that Mahler's pseudovaluations [8] do not exhaust all possible topologies of, for instance, the rational number field; a topology which is the "direct sum" in Mahler's sense of infinitely many valuations would be an example of a topology in which we cannot have $|a b| \leqq|a||b|$ (we define $|a|=\sum_{p} 2^{-p}|a|_{p}$ ).

In conclusion we wish to point out that the results contained in A. H. Frink's paper [9] can be extended to the present paper. The generalized distance function can be assumed to have values in any uniform space $V$ and to satisfy the following conditions: II (1') $(s, t) \neq u_{0}$ for some $u_{0} \in V ;(s, t)=u_{0}$ if and only if $s=t$. II (2) As above. II (3') For every neighborhood $N$ of $u_{0}$ and for every $s \in S$ there is a neighborhood $M$ of $u_{0}$ such that $(s, r) \in M,(r, t) \in M$ imply $(s, t) \in N . S$ is then topologized as above: $U_{N}(s)=\left[t ;(s, t) \in N\left(u_{0}\right)\right]$. Using the generalized metric of $V$ we can use its components as pseudometrics of $S$, and by the arguments of [9] we can prove $S$ to 
be a generalized metric space. This allows us somewhat to modify Weil's postulates for uniform spaces, where (cf. [6, p. 7]) we replace $U_{\text {III }}$ by requiring $p \in V(q)$ if and only if $q \in V(p)$; the index set $I$ is ordered by inclusion of the corresponding neighborhoods; given $s \in S$, $i \in I$ there exists $j=j(s, i) \in I$ such that $s \in V_{j}(r), r \in V_{j}(t)$ imply $s \in V_{i}(t)$. We prove $S$ to be a uniform space by introducing into it a generalized distance function based on the set $I$ and defining $(s, t)_{i}=1$ if $s$ is not in $V_{i}(t)$ and 0 elsewhere.

\section{BIBLIOGRAPHY}

1. G. B. Price, $A$ generalization of a metric space with applications to spaces whose elements are sets, Amer. J. Math. vol. 63 (1941) pp. 46-56.

2. W. Krull, Allgemeine Bewertungstheorie, J. Reine Angew. Math. vol. 167 (1931) pp. 160-196.

3. D. H. Hyers, Pseudo-normed linear spaces and abelian groups, Duke Math. J. vol. 5 (1939) pp. 628-634.

4. A. H. Clifford, Partially ordered abelian groups, Ann. of Math. vol. 41 (1940) pp. $465-473$.

5. H. Hahn, Ueber die nichtarchimedischen Groessensysteme, Sitzungsberichte der Mathematischen-Naturwissenschaftliche Klasse der Kaiserlichen Akademie der Wissenschaften, Vienna vol. IIa 116 (1907) pp. 601-655.

6. A. Weil, Sur les espaces d structure uniforme et sur la topologie generale, Paris, Hermann, 1938.

7. S. Kakutani, Ueber die Metrisierung der topologischen Gruppen, Proc. Imp. Acad. Tokyo vol. 12 (1936) pp. 82-84.

8. K. Mahler, Ueber Pseudobewertungen II, Acta Math. vol. 67 (1936) pp. 51-80.

9. A. H. Frink, Distance functions and the metrization problem, Bull. Amer. Math. Soc. vol. 43 (1937) pp. 133-142.

Cornell University 